\title{
Caracterización de preguntas en libros de texto de Ciencias Naturales en Educación Primaria chilena
}

\author{
Alejandro Sepúlveda ${ }^{1}$ \\ http://orcid.org/0000-0002-5033-8400 \\ Andrea Minte ${ }^{1}$ \\ http://orcid.org/0000-0002-8720-692X \\ Danilo Díaz-Levicoy² \\ http://orcid.org/0000-0001-8371-7899
}

\section{Resumen}

La formulación de preguntas es una actividad frecuente en los procesos de enseñanza y de aprendizaje. Su promoción es una tarea relevante en las aulas tanto en contextos orales como escritos. De acuerdo con lo anterior, el presente trabajo da cuenta de los resultados obtenidos en una investigación de las dimensiones del proceso cognitivo que demandan las preguntas formuladas en los libros de texto de Ciencias Naturales para estudiantes de Educación Primaria chilena. Se trató de una investigación con método cuantitativo, en la cual se clasificaron los tipos de preguntas de acuerdo a la dimensión del proceso cognitivo, que demandan a los estudiantes. Se utilizó como base la taxonomía de Anderson y Krathwohl (2001), con la finalidad de evaluar las preguntas presentes en los libros de texto actuales, específicamente, en el contenido y en las actividades didácticas propuestas. Se trabajó con el total de preguntas correspondiente a 1256. Se analizaron estadísticamente con el software SPSS (15.0). Entre los hallazgos se puede señalar que las preguntas contenidas en los libros de texto de Ciencias Naturales promueven, mayoritariamente, la dimensión más elemental de la taxonomía, recordar información y, en menor medida, las dimensiones cognitivas superiores: crear, evaluar y aplicar. Cabe destacar que no se constatan diferencias estadísticamente significativas entre las demandas de las preguntas formuladas en los textos de los diferentes cursos estudiados.

\section{Palabras clave}

Preguntas - Demanda cognitiva - Libros de texto - Ciencias naturales.

1- Universidad de Los Lagos, Osorno, Chile. Contactos: asepulve@ulagos.cl; andrea.minte@ulagos.cl.

2- Universidad Católica del Maule, Talca, Chile. Contacto: dddiaz01@hotmail.com. 


\title{
Characterization of questions in textbooks of Natural Science in Chilean Primary Education
}

\begin{abstract}
Asking questions is a frequent activity in the teaching and learning processes. Its promotion is an important task in classrooms both in oral and written contexts. In accordance with the above, this work reports the results obtained in an investigation of the dimensions of the cognitive process demanded by the questions formulated in Natural Science textbooks for Chilean Primary Education students. It was an investigation with a quantitative method, in which the types of questions were classified according to the dimension of the cognitive process the students were demanded. The taxonomy of Anderson and Krathwohl (2001) was used as a basis, in order to evaluate the questions in current textbooks, specifically, in content and in the proposed didactic activities. We worked with the total of questions corresponding to 1256. Among the findings, a highlight was that the questions contained in Natural Science textbooks promote the most elemental dimension of taxonomy, recalling information and, to a lesser extent, the higher cognitive dimensions: creating, evaluating and applying. It should be noted that there are no statistically significant differences among the demands of the questions asked in the texts in the different courses studied.
\end{abstract}

\section{Keywords}

Questions - Cognitive demand - Textbooks - Natural science.

\section{Introducción}

La construcción de nuevos conocimientos científicos se inicia con una buena pregunta. De esta forma, se puede señalar que las preguntas constituyen la base del conocimiento científico y, por tanto, de los avances de la ciencia. Para Márquez y Roca (2006) identificar y plantearse preguntas es parte de hacer ciencia y es necesario enseñar a los estudiantes a formularlas para iniciarlos en la cultura científica.

Por tanto, si se pretende que la enseñanza sea un proceso cognitivo y concordante con las actividades que realizan los científicos, es importante que los profesores formulen preguntas de alto nivel cognitivo (CHINN; MALHOTRA, 2002; GIL-PÉREZ et al., 1991; HOFER; PINTRICH, 1997). Así como en la ciencia, la formulación de preguntas es también una actividad fundamental en la educación. Las preguntas son determinantes en los procesos de enseñanza y de aprendizaje en las aulas, en los libros de texto, en las pruebas. Se considera que las intervenciones para mejorar el proceso de enseñanza de los estudiantes y su calidad deberían empezar por cambiar las preguntas que se formulan tanto por parte de los profesores (JUANDÓ; PÉREZ-CABANÍ, 2010; PÉREZ-CABANÍ; CARRETEROTORRES, 2003; PÉREZ-CABANÍ; CARRETERO-TORRES; JUANDÓ, 2009) como las que se encuentran en los libros de texto. 
Las preguntas se consideran un aspecto central en el desarrollo del proceso cognitivo en todos los niveles educativos y en diferentes escenarios. Diferentes estudios de Graesser y otros autores distinguen preguntas de razonamiento superficial, intermedio y profundo (GRAESSER; PERSON, 1994; GRAESSER; PERSON; HUBER, 1992; GRAESSER; OZURU; SULLINS, 2009). Las buenas preguntas se caracterizan por ser estimulantes para activar los conocimientos previos y sirven para establecer causalidad y otros procesos cognitivos superiores, tales como comparaciones entre fenómenos u objetos. Esto es particularmente importante en la enseñanza de las Ciencias Naturales.

Por otra parte, Vidal-Abarca, Gilabert y Rouet (2005) encontraron que las preguntas intercaladas en los libros de texto de ciencias poseen un efecto positivo en el aprendizaje de fenómenos científicos. Además, lograron establecer que, presentadas de esta forma, las preguntas, poseen un doble efecto: 1) permiten contener con mayor profundidad ciertos contenidos y desarrollar procesos mentales valiosos para la ciencia (explicar fenómenos, realizar inferencias, razonar deductiva e inductivamente); y 2) evalúan si la información ha sido comprendida. Adicionalmente, estos autores destacan la importancia de las preguntas intercaladas con textos expositivos, lo cual tiene un efecto positivo en el aprendizaje de la ciencia.

El desarrollo del pensamiento científico y la comprensión profunda han sido temas investigados por Chin y Osborne (2008). Chin (2004) ha propuesto recomendaciones que permiten una adecuada formulación de preguntas para el desarrollo del pensamiento profundo en Biología. Del mismo modo, destacan los estudios de Oliveira (2010) y Van Zee (2000) acerca del desarrollo del razonamiento científico. Se sostiene que las preguntas de alto nivel de elaboración generan hipótesis y permiten recoger información relacionada con ella, es decir, van más allá de lo señalado por los textos (GRAY, 1993; HALPERN, 1994). Estos tipos de procesos se corresponden con los de pensamiento científico (CHIN; OSBORNE, 2008).

Chin y Chia (2004) revisaron taxonomías de preguntas formuladas en el ámbito de la enseñanza y del aprendizaje de las Ciencias Naturales, considerando el contexto de aula y la docencia en laboratorios. En cuanto al tipo de información que una pregunta demanda, existen diversas tipologías, a saber: preguntas cerradas y abiertas, generales y específicas, predictivas, explicativas, comparaciones, conexiones, sobre hechos y evaluativas.

Graesser, Person y Huber (1992) presentaron una taxonomía de dieciocho categorías, basada en el tipo de información que se podía obtener de la pregunta. Entre éstas se encuentran: compleción de conceptos, especificación de características, verificación, cuantificación, comparación, antecedente causal, interpretación, consecuente causal, juicio, petición, disyunción, orientación a la meta, instrumental-procedimental, expectativa, aserción, definición, ejemplo y posibilitación. Los autores señalan que estas categorías se pueden superponer, es decir, pueden corresponder a más de una categoría, por lo que se contabilizarán tantas veces aparezcan. Ello depende del contenido y de la demanda de la pregunta.

La importancia de formular preguntas, de acuerdo con Trillo (2005), radica en que evocan algún camino para su resolución y permiten conectar el contenido con otros temas, además de las propias experiencias. Sostiene que los estudiantes deben utilizar los conocimientos previos y desarrollar procesos de deliberación para mejorar la comprensión. 
Respecto de la calidad de la pregunta, no existirian preguntas buenas o malas. Las preguntas que pretenden la reproducción de contenidos, así como las elaborativas, tienen valor en sí mismas. Poseen finalidades diferentes. Ambas son valiosas, ya que las menos elaborativas deben preceder a aquellas para fijar el conocimiento y poder desarrollar competencias cognitivas de orden superior. En la literatura especializada se encuentran algunas definiciones de preguntas de calidad. Para Marbach-Ad y Sokolove (2000) las buenas preguntas son las de investigación; Graesser, Ozuru y Sullins (2009) sostienen que son las preguntas que apoyan el aprendizaje, y según Scardamalia y Bereiter (1992) las buenas preguntas son aquellas que poseen valor educativo.

En síntesis, las preguntas son significativas tanto para desarrollar diferentes niveles de competencias cognitivas, como para potenciar el proceso de enseñanza y de aprendizaje, así como para el progreso de la ciencia.

Por este motivo se estudiaron las preguntas contenidas en los libros de texto de Ciencias Naturales. Éstos “[...] permiten que los estudiantes accedan al conocimiento de una disciplina, existiendo una coherencia entre el contenido que describe y los objetivos mencionados en las directrices curriculares" (DÍAZ-LEVICOY et al., 2016, p. 93).

El libro de texto sigue siendo uno de los recursos didácticos más importantes y de mayor tradición en las aulas. Aunque existan críticas a estos y la tecnología haya invadido las escuelas y se le considere un elemento deformante, impositivo y moralizante (COLÁS, 1989), el libro de texto posee una inquebrantable vigencia. De esta forma, este recurso se mantiene como producto de interés científico. Entre las cuatro características esenciales que Colás (1989) distingue, se pueden sintetizar las siguientes: 1) es un material eficaz; 2) es asequible; 3) permite niveles de abstracción que otros medios no logran, y 4) aporta experiencias vicarias, abstractas y mediatas que favorecen el aprendizaje.

La importancia de estudiar las preguntas en los libros de texto se basa en que hasta el momento ningún otro medio ha podido superar la primacía de este recurso didáctico (MARTÍNEZ-BONAFÉ, 2002; TYNER, 1993; CHOPPIN, 2001; COLÁS, 1989; COSTA, 1990). $\mathrm{Su}$ utilización se debe, fundamentalmente, a que orientan y dirigen actividades tanto de docentes como de estudiantes (CAMPANARIO; OTERO, 2000). Permiten asegurar la equidad y calidad (CHILE, 2015) y, además, materializan la cultura y los saberes considerados necesarios para ser transmitidos (OCCELLI; VALEIRAS, 2013).

En Chile, la política pública establece la distribución de libros de texto de forma masiva, gratuita y sistemática. Corresponde a las asignaturas prioritarias definidas por el Estado, entre las cuales se encuentran los libros de texto de Ciencias Naturales. Los destinatarios son los estudiantes, sus familias y también los profesores, especialmente para aquellas personas que requieren apoyo tanto por su lejanía geográfica como por principios de equidad y acceso a la cultura (CHILE, 2015).

Para el Ministerio de Educación los libros de texto son una herramienta clave en el fomento de habilidades, conocimientos y actitudes. Tienen por finalidad mejorar la calidad de los aprendizajes (CHILE, 2015). Por estas razones, se distribuye anualmente un gran número de ejemplares, el cual ha aumentado un 90\% en casi 30 años. Esto se observa en la Tabla 1. 
Tabla 1- Entrega de libros de texto, comportamientos y evolución (1990-2016)

\begin{tabular}{ccccccc}
\hline \multirow{2}{*}{ Años } & $\vdots$ & $\begin{array}{c}\text { Cantidad de textos } \\
\text { (en miles de ejemplares) }\end{array}$ & Nivel de cobertura & & Nivel educacional \\
\hline 1990 & 1920 & 7500 & $85 \%$ & Básica \\
\hline 2000 & 15253 & $100 \%$ & Básica y media \\
\hline 2010 & 17500 & $100 \%$ & Parvularia, Básica y Media \\
\hline 2015 & 17780 & $100 \%$ & Parvularia, Básica y Media \\
\hline 2016 & $\vdots$ & $100 \%$ & Parvularia, Básica y Media \\
\hline
\end{tabular}

Fuente: Ministerio de Educación, Chile (CHILE, 2015).

En 2012 el Ministerio de Educación chileno solicitó a una empresa externa la evaluación de la política de libros de texto, con el fin de valorar la política de implementación paulatina de estos recursos los últimos siete años (THIBAUT; MEDRANO; JIMÉNEZ, 2012). Las conclusiones de este estudio revelan que: 1) los estudiantes consideran importante la distribución gratuita de estos textos, debido a que los acerca al conocimiento, permite encontrar información, estudiar, realizar tareas, entretenerse; 2) los profesores señalan que facilitan la labor docente, profundizan conocimientos, complementan contenidos, abren nuevas posibilidades de consolidación de conocimientos, permiten realizar las actividades propuestas en ellos; 3) los libros de texto que ofrecen mayor grado de comprensión corresponde a los de las Ciencias Naturales; 4) este recurso sigue sub utilizándose al continuar con el uso del dictado en clases. Ello se evidencia en lo expresado en el informe: "[...] para copiar la materia en el cuaderno, el libro de ciencias naturales es el más nombrado por los estudiantes" (THIBAUT; MEDRANO; JIMÉNEZ, 2012, p. 210); 5) los docentes manifiestan que el lenguaje empleado en los libros de texto presenta dificultades, ya que se considera complicado y alejado de la realidad. Es decir, los estudiantes no están familiarizados con el uso formal de la lengua materna; 6) la mayoría de los docentes sostiene que los libros de texto son importantes como de apoyo a su labor, sin embargo, señalan que éstos adolecen de una orientación práctica que les permita desarrollar “[...] el pensamiento crítico, creador y divergente en la búsqueda de resolución de problemas" (THIBAUT; MEDRANO; JIMÉNEZ, 2012, p. 211).

La cita precedente refrenda la idea de que los libros de texto no promueven el desarrollo de procesos cognitivos de orden superior. Sin embargo, no pierden vigencia, ya que constituyen un recurso significativo, el cual no ha podido ser desplazado como recurso didáctico relevante en los procesos de enseñanza y aprendizaje (COLÁS, 1989; OTER0; GRECA, 2004), a pesar de la predominancia de la multimedia y lo multimodal.

Respecto de los libros de texto de Ciencias Naturales aludidos en el señalado estudio, se señala que son los más adecuados en la presentación de los contenidos y también son muy valorados desde el punto de vista metodológico (THIBAUT; MEDRANO; JIMÉNEZ, 2012), no obstante, no hay mención acerca del tema de las preguntas. Investigaciones precedentes abordaron la pertinencia, la calidad, el contenido, las actividades, el lenguaje utilizado, entre otros elementos, sin embargo, el tema de las preguntas no figura entre las variables consideradas en la evaluación realizada. 
En la presente investigación se analizaron las preguntas contenidas en los libros de texto y se recurrió a la taxonomía de Anderson y Krathwohl (2001), emanada de la taxonomía de objetivos educativos de Bloom (BLOOM et al., 1956). La taxonomía de Bloom se convirtió en un elemento fundamental para estructurar y comprender el proceso de enseñanza y de aprendizaje, el cual adscribe a uno de estos tres dominios: 1) cognitivo: en el cual se procesa información, conocimientos y habilidades mentales; 2) afectivo: alude a actitudes y sentimientos involucrados en el proceso; y 3) psicomotor: se refiere a habilidades motoras, manuales o físicas.

Anderson y Krathwohl (2001) introdujeron algunos ajustes a la taxonomía. Los principales cambios consistieron en el reemplazo de los sustantivos de la propuesta original de Bloom a verbos, con la finalidad de destacar las acciones correspondientes a cada categoría. Otra innovación fue la ampliación de la categoría de síntesis, la cual relacionaron con la creación. Finalmente, modificaron la secuencia de las categorías, quedando en grados de complejidad creciente: recordar, comprender, aplicar, analizar, evaluar y crear. La taxonomía fue utilizada en el presente estudio, debido a que posee una alta confiabilidad interjueces, según lo demostrado por Näsström y Henriksson (2008).

De acuerdo con las consideraciones anteriores, el objetivo general de la investigación fue determinar la dimensión del proceso cognitivo que promueven las preguntas formuladas en los libros de texto chilenos de Educación Primaria de Ciencias Naturales.

\section{Método}

Se trata de una investigación con método cuantitativo, de tipo descriptiva, en la cual se trabajó el total de preguntas formuladas en los cuatro libros de texto de Ciencias Naturales para la Educación Primaria chilena (11-14 años), publicados en 2019 (ver Cuadro 1). Se trata de los textos oficiales, distribuidos al 99,5\% de los establecimientos educacionales públicos del país. Los criterios de selección fueron: 1) se utilizan actualmente en ese nivel de enseñanza; 2) son los textos oficiales licitados por el Ministerio de Educación; 3) poseen la mayor cobertura geográfica; 4) su distribución es gratuita; y 5) son de reciente publicación.

Cuadro 1 - Corpus de libros de texto de Ciencias Naturales de Educación Primaria

\begin{tabular}{|l|l|c|}
\hline \multicolumn{1}{|c|}{ Autores } & \multicolumn{1}{|c|}{ Titulo } & \multicolumn{1}{c|}{ Editorial } \\
\hline Valdés. P. y Rojas, M. & Ciencias Naturales. Texto del estudiante. Quinto año. & Crecer pensando \\
\hline Morales, K.; Ortiz, P. y Valdebenito, S. & Ciencias Naturales. Texto del estudiante. Sexto año. & SM \\
\hline Romero, C.; Tobar, C y Muñoz, D. & Ciencias Naturales. Texto del estudiante Séptimo Básico & SM \\
\hline Campbell, E. & $\begin{array}{l}\text { Ciencias Naturales. Texto del estudiante. Octavo año de Enseñanza } \\
\text { Básico }\end{array}$ & SM \\
\hline
\end{tabular}

Fuente: Elaborado por los autores.

Las preguntas contenidas en cada uno de los libros de texto, correspondientes a 1256 preguntas, constituyeron el objeto de estudio. La cantidad de preguntas se desglosa por curso y por unidad temática en el Tabla 2. 
Tabla 2 - Cantidad de preguntas por curso y por unidades temáticas

\begin{tabular}{|c|c|c|c|c|c|c|c|c|}
\hline \multicolumn{9}{|c|}{ Unidades } \\
\hline & & $1 \mathrm{ra}$ & $2 \mathrm{da}$ & $3 r a$ & 4 ta & 5 ta & 6 ta & Total Preguntas \\
\hline \multirow{4}{*}{$\begin{array}{l}\text { क्ठ } \\
\text { 站 }\end{array}$} & Quinto & 82 & 57 & 79 & 66 & - & - & 284 \\
\hline & Sexto & 100 & 93 & 85 & 69 & - & - & 347 \\
\hline & Séptimo & 70 & 54 & 46 & 58 & 58 & 44 & 330 \\
\hline & Octavo & 64 & 59 & 57 & 54 & 61 & - & 295 \\
\hline \multicolumn{8}{|c|}{ Total } & 1256 \\
\hline
\end{tabular}

Fuente: Elaborado por los autores.

Las preguntas fueron analizadas y categorizadas a partir de la taxonomía de Anderson y Krathwohl, para quienes “[...] los procesos cognitivos son vías con las que los estudiantes pueden involucrarse activamente en la construcción de aprendizajes significativos” (2001, p. 65).

Para efectos de esta investigación, se elaboró una matriz con los niveles de razonamiento y las dimensiones cognitivas de la taxonomía señalada, con ejemplos de verbos correspondientes a cada dimensión, lo cual se presenta en el Cuadro 2.

Cuadro 2 - Dimensiones, proceso cognitivo y verbos

\begin{tabular}{|c|c|c|}
\hline Nivel de razonamiento & Dimensiones del proceso & Procesos cognitivos y verbos \\
\hline \multirow{2}{*}{ Básico } & \multirow{2}{*}{$\begin{array}{l}\text { 1. Recordar: recuperar de la memoria a largo plazo el conocimiento } \\
\text { pertinente }\end{array}$} & 1.1. Reconocer \\
\hline & & 1.2. Evocar \\
\hline \multirow{7}{*}{ Intermedio } & \multirow{7}{*}{$\begin{array}{l}\text { 2. Comprender: construir significados a partir de mensajes } \\
\text { obstruccionales, incluyendo la comunicación oral escrita y gráfica }\end{array}$} & 2.1. Interpretar \\
\hline & & 2.2. Ejemplificar \\
\hline & & 2.3. Clasificar \\
\hline & & 2.4. Resumir \\
\hline & & 2.5. Inferir \\
\hline & & 2.6. Comparar \\
\hline & & 2.7. Explicar \\
\hline \multirow{10}{*}{ Profundo } & \multirow{2}{*}{$\begin{array}{l}\text { 3. Aplicar: llevar a cabo o utilizar un procedimiento de una } \\
\text { situación dada. }\end{array}$} & 3.1. Ejecutar \\
\hline & & 3.2. Implantar \\
\hline & \multirow{3}{*}{$\begin{array}{l}\text { 4. Analizar: descomponer el material en sus partes integrantes y } \\
\text { determinar de qué manera se relacionan unas con otras y con una } \\
\text { estructura o propósito general }\end{array}$} & 4.1. Diferenciar \\
\hline & & 4.2. Organizar \\
\hline & & 4.3. Atribuir \\
\hline & \multirow{2}{*}{ 5. Evaluar: emitir juicios basados en criterios y normas } & 5.1. Comprobar \\
\hline & & 5.2. Criticar \\
\hline & \multirow{3}{*}{$\begin{array}{l}\text { 6. Crear: reunir elementos para formar un todo coherente } 0 \\
\text { funcional; reorganizar elementos en un nuevo modelo o estructura }\end{array}$} & 6.1. Generar \\
\hline & & 6.2. Planificar \\
\hline & & 6.3. Producir \\
\hline
\end{tabular}

Fuente: Adaptado de Anderson y Krathwohl (2001) 
Con el fin de velar por la objetividad en el análisis de las preguntas y su clasificación de acuerdo al nivel cognitivo, se recurrió a cuatro jueces, quienes trabajaron de forma conjunta. Cada uno de los expertos valoró y clasificó todas las preguntas. Se consideró el criterio de coincidencia del 75\%. En los veinte casos en que no hubo concordancia, se sometió a consideración a un quinto juez. De esta forma, se pudo discriminar y se logró completo acuerdo respecto de la dimensión del proceso cognitivo que la pregunta demandaba a los estudiantes, de acuerdo con la taxonomía.

Ejemplos de preguntas, que tuvieron que ser re-clasificadas por no haber coincidido en primera instancia en las categorías preestablecidas, fueron los siguientes. ¿A qué tipo de consumidor corresponde la nutria? (Libro de texto de Ciencias Naturales, $6^{\circ}$ año, $\mathrm{p}$. 96) y ¿De qué partículas se componen los granos de arena? (Libro de texto de Ciencias Naturales, $8^{\circ}$ año, p. 146). Estos y otros casos se resolvieron eficazmente con la decisión de un quinto experto. Esta modalidad permitió el alcanzar la objetividad esperada.

Se aplicaron cuatro funciones estadísticas del software estadístico SPSS versión 15.0. El primer test fue la prueba no paramétrica $H$ de Kruskal-Wallis. Se seleccionó esta función debido a la naturaleza de la variable, que es categórica ordinal. Otra razón de la selección de esta prueba es que se trata de cuatro muestras independientes. Se combinaron todos los datos de la muestra (preguntas), luego se ordenaron de menor a mayor en la variable nivel de razonamiento, se asignó el rango correspondiente, se sumaron en cada grupo por separado y se obtuvo la media de rangos.

También se realizó el análisis de la variable dimensión del proceso cognitivo de las preguntas formuladas en los libros de texto, según el curso en el cual se plantean. Luego se realizó un análisis respecto de las unidades temáticas contenidas en los mismos libros.

El segundo test consistió en la aplicación de la prueba no paramétrica $H$ de KruskalWallis, con la finalidad de estudiar la existencia de diferencias estadísticamente significativas en el nivel de razonamiento de las preguntas y las unidades temáticas. Se estudiaron las diferencias entre grupos de la variable dimensión del proceso cognitivo de las preguntas contenidas en los libros de texto y la unidad temática en las cuales se presentan.

En el contexto del tercer test aplicado, se utilizó el Coeficiente de Correlación de Spearman, con el objeto de estudiar la existencia de asociación entre la dimensión del proceso cognitivo de las preguntas formuladas en los textos y el curso en el cual se encuentran. Después de probar la existencia de esta asociación, se midió la fuerza de intensidad de la misma, según el criterio propuesto por Hernández, Fernández y Baptista (2010).

El cuarto test aplicado fue la prueba estadística Chi Cuadrado con el software SPSS. Se asoció la dimensión del proceso cognitivo con las unidades temáticas contenidas en el total de libros de texto estudiados.

\section{Resultados}

En esta parte se presenta el análisis estadístico al cual se sometieron las preguntas del estudio. En primer lugar, se muestran las frecuencias y porcentajes de preguntas con el correspondiente nivel de razonamiento y la dimensión del proceso cognitivo. Se responde en términos estadísticos la siguiente interrogante ¿el nivel de razonamiento y dimensión 
del proceso cognitivo de las preguntas formuladas en los libros de texto de Ciencias Naturales es diferente, según el curso y la unidad en los cuales se plantean?

La Figura 1 da cuenta de que las preguntas en los libros de texto de Ciencias Naturales se agrupan en el nivel de razonamiento básico, el cual corresponde al 44,3 $\%$. Se incluye el uso de la memoria, la definición de conceptos, el recuerdo de nombres, fechas y datos. Ejemplos: a) "La piel humana, ¿De qué sistema forma parte?” (Ciencias Naturales, $5^{\circ}$ año, p. 93); b) "Menciona tres ejemplos de organismos que realicen fotosíntesis” (Libro de texto Ciencias Naturales, $6^{\circ}$ año, p. 85).

El nivel intermedio implica identificar las ideas fundamentales de un mensaje, justificar ideas, ejemplificar corresponde al 20,9\% de las preguntas. Ejemplos: a) "¿Cuáles podrían ser las razones que motivan a algunas mujeres a donar sus ovocitos?" (Ciencias Naturales, $6^{\circ}$ año, p. 18); b) “¿Qué diferencias y similitudes distinguen en las enfermedades que se presentan?” (Ciencias Naturales, $5^{\circ}$ año, p. 126).

En cambio, el nivel de razonamiento superior comprende la construcción de significados, interpretar mensajes, clasificar, inferir, aplicar, proponer y crear. Esto se promueve en el 34, 7\% de las preguntas en los libros de texto de la muestra. Ejemplos: a) "¿Qué actividades humanas crees que han contribuido al incremento del efecto invernadero? Argumenta” (Ciencias Naturales, $7^{\circ}$ año, p. 183); “¿Qué relación se puede establecer entre la actividad física y el estado de salud de las personas?" (Ciencias Naturales, $6^{\circ}$ año, p. 35).

Figura 1 - Porcentaje de preguntas, según el nivel de razonamiento, en libros de texto de Ciencias Naturales

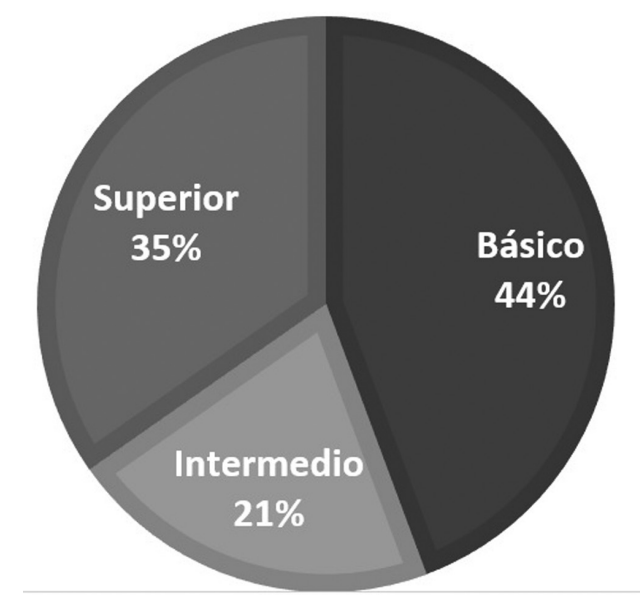

Fuente: Elaborado por los autores.

\section{Análisis descriptivo del nivel de razonamiento básico e intermedio de las preguntas}

De un total de 1.256 preguntas enunciadas en los libros de texto de Ciencias Naturales, cursos: $5^{\circ}, 6^{\circ}, 7^{\circ}$ y $8^{\circ}$, se precisa que: 
- 557 preguntas están asociadas al nivel de razonamiento básico, de las cuales 122 se agrupan en quinto básico, 157 en sexto, 156 en séptimo y 122 en octavo año. Respecto del nivel de razonamiento intermedio, 263 preguntas se distribuyen en él. Se desglosan en 64 para quinto, 61 en sexto, 72 en séptimo y 66 en octavo año.

- Los guarismos restantes se interpretan de forma análoga y se exponen en la Tabla 3.

Tabla 3 - Frecuencia y porcentaje de nivel de razonamiento, según cursos de Educación Primaria

\begin{tabular}{cccccc}
\hline Nivel de razonamiento & $5 \% \%$ & $6 \% \%$ & $7 \% \%$ & $8 \% \%$ & Total/ \% \\
\hline Básico & $122(43)$ & $157(45,2)$ & $156(47,3)$ & $122(41,4)$ & $557(44,3)$ \\
Intermedio & $64(22,5)$ & $61(17,6)$ & $72(21,8)$ & $66(22,4)$ & $263(20,9)$ \\
\hline
\end{tabular}

Fuente: Elaborado por los autores.

Con el propósito de determinar diferencias estadísticas entre el nivel de razonamiento de las preguntas contenidas con los libros de texto de Ciencias Naturales y el curso en el cual se formulan, se recurrió al valor del test de $H$ Kruskal-Wallis. El resultado fue $p=0,421$ lo que significa que no se observan diferencias sustantivas entre las preguntas expuestas y el curso en el cual se plantean (Tabla 4).

Tabla 4 - Cálculo H de Kruskal-Wallis

\begin{tabular}{|c|c|}
\hline & Estadísticos de prueba ${ }^{\mathrm{a}, \mathrm{b}}$ \\
\hline & Nivel cognitivo \\
\hline Chi-cuadrado & 2,814 \\
\hline gl & 3 \\
\hline Sig. asintótica &, 421 \\
\hline
\end{tabular}

a. Prueba de Kruskal Wallis; b. Variable de agrupación: curso

El grado de significancia utilizado fue del $5 \%$.

Sig. Asintótica: nivel de significación frontera para rechazar o aceptar la hipótesis nula con los datos obtenidos en la muestra.

Fuente: Elaborado por los autores.

\section{Análisis descriptivo de la dimensión del proceso cognitivo superior promovidas por las preguntas}

En relación a las dimensiones cognitivas superiores (aplicar, analizar, evaluar y crear), de las 1256 preguntas formuladas en los libros de texto de Educación Primaria se observó lo siguiente:

- 436 preguntas se ubican en la dimensión del proceso cognitivo superior. Se reconocen 98 preguntas en quinto año, 129 en sexto, 102 en séptimo y 107 en octavo año. 
- Respecto de las diversas dimensiones del proceso cognitivo de orden superior se constató lo siguiente: 127 preguntas se evalúan como de aplicación, 112 de análisis, 124 de evaluación y 73 preguntas de creación.

Desagregadas las diferentes dimensiones del proceso cognitivo por curso (quinto al octavo año), se observa una disminución de la cantidad de preguntas en la medida que se asocian a niveles superiores. En concreto, la cantidad de preguntas formuladas en todos los cursos es menor en la dimensión crear. En términos comparativos entre cursos la tendencia es a la baja.

Porcentualmente no se constatan diferencias estadísticamente significativas entre la dimensión del proceso cognitivo de las preguntas formuladas en los libros de texto de Ciencias Naturales y el curso en el cual se plantean (Tabla 5).

Tabla 5 - Frecuencia y porcentaje de las dimensiones del proceso cognitivo, según cursos de Educación Primaria

\begin{tabular}{|c|c|c|c|c|c|}
\hline Nivel cognitivo & $5 \% \%$ & $6 \% \%$ & $7 \% \%$ & $8 \% \%$ & Total / \% \\
\hline Aplicar & $30(10,6)$ & $31(8,9)$ & $32(9,7)$ & $34(11,5)$ & $127(10,1)$ \\
\hline Analizar & $22(7,7)$ & $34(9,8)$ & $29(8,8)$ & $27(9,2)$ & $112(8,9)$ \\
\hline Evaluar & $30(10,6)$ & $37(10,7)$ & $28(8,5)$ & $29(9,8)$ & $124(9,9)$ \\
\hline Crear & $16(5,6)$ & $27(7,8)$ & $13(3,9)$ & $17(5,8)$ & $73(5,8)$ \\
\hline
\end{tabular}

Fuente: Elaborado por los autores.

En el análisis de la dimensión del proceso cognitivo también se aplicó el test no paramétrico denominado $H$ de Kruskal-Wallis (Tabla 6). En este caso, el $p$-valor (H KruskalWallis, $p=0,342$ ) muestra que no existen diferencias estadísticamente significativas en la dimensión del proceso cognitivo de preguntas formuladas en los libros de texto de Ciencias Naturales y los diferentes cursos en los cuales se registran estas preguntas.

Tabla 6 - Cálculo H de Kruskal-Wallis

Estadísticos de prueba ${ }^{a, b}$

\begin{tabular}{cc}
\hline & Proceso cognitivo \\
\cline { 2 - 2 } Chi-cuadrado & 3,343 \\
Slg. asintótica &, 3 \\
a. Prueba de Kruskal Wallis; b. Variable de agrupación: curso
\end{tabular}

El grado de significancia utilizado fue del $5 \%$.

Sig. Asintótica: nivel de significación frontera para rechazar o aceptar la hipótesis nula con los datos obtenidos en la muestra.

Fuente: Elaborado por los autores. 
La Figura 2 muestra la dimensión del proceso cognitivo que promueven las preguntas de acuerdo con las unidades temáticas consideradas en los libros de texto de Ciencias Naturales de los cursos de $5^{\circ}$ a $8^{\circ}$ año.

Se observa que en las unidades temáticas declaradas en los cursos de quinto a octavo año básico, se constata la formulación de preguntas correspondientes a todas las dimensiones del proceso cognitivo. La dimensión recordar es la que, mayoritariamente, está presente en la totalidad de las unidades. No se advierten diferencias estadísticamente importantes entre la dimensión del proceso cognitivo de la pregunta y la unidad temática. No se constata la presencia de preguntas de una determinada dimensión del proceso cognitivo en una unidad temática.

Figura 2 - Cantidad de preguntas en unidades temáticas por curso y dimensión del proceso cognitivo

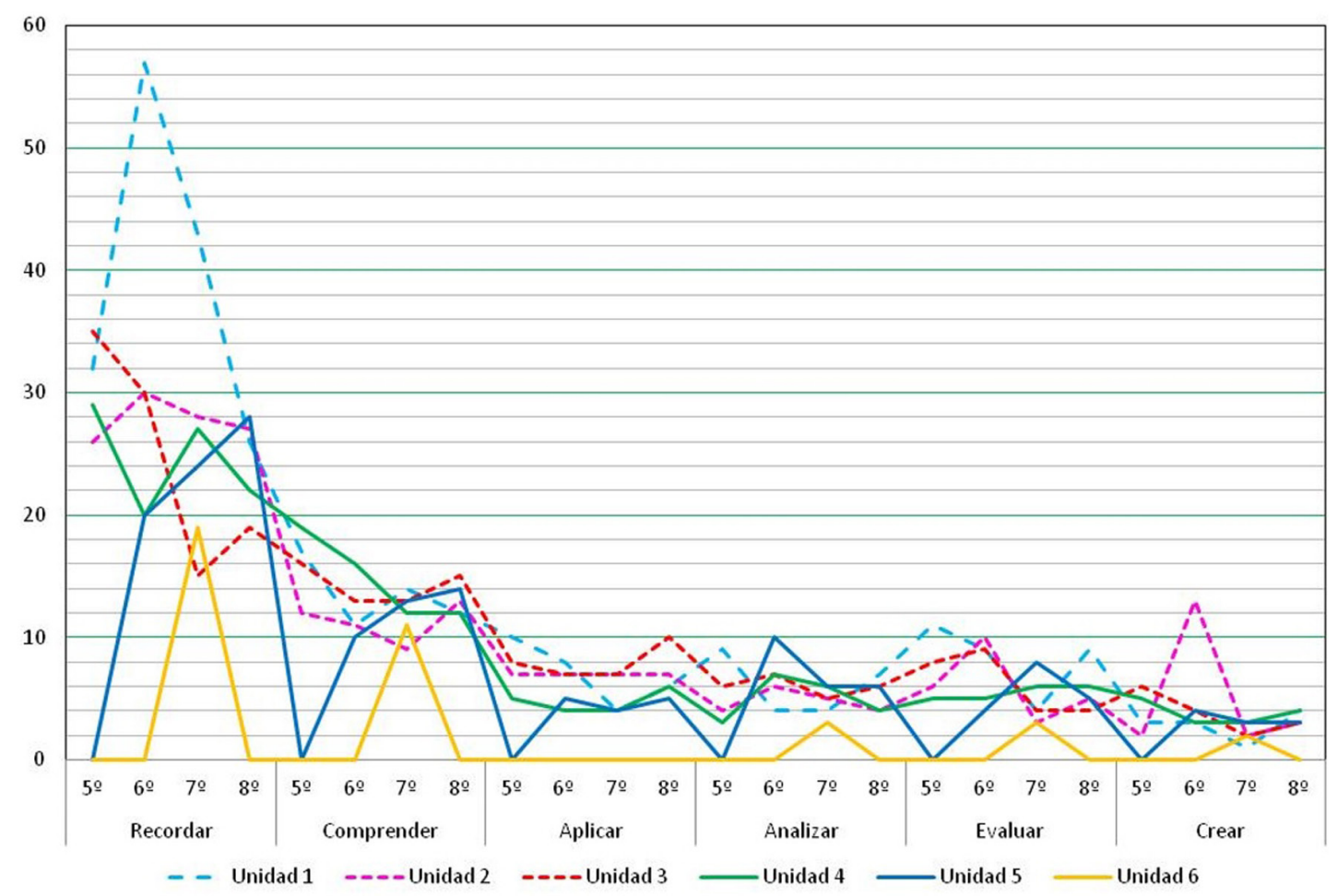

Fuente: Elaborado por los autores.

\section{Discusión y conclusiones}

Las preguntas formuladas en los libros de texto analizados promueven el razonamiento básico en un 44\% (el nivel recordar), 22,5\% en nivel intermedio (comprender) y demandan, de las demás dimensiones (aplicar, analizar, evaluar y crear) el 34,5\%. La dimensión del proceso cognitivo de orden superior (crear) ocupa solo el 5,6\%. 
En un estudio similar (MENESES; MONTENEGRO; RUIZ, 2013) se sostiene que los procesos cognitivos promovidos para lograr aprendizajes en Ciencias Naturales en Chile se concentran en las categorias de recordar (26,7\%) y comprender (49\%). En una investigación comparada, Canadá y Singapur presentan valores más bajos en estas categorías, pero destinan un porcentaje más elevado $(24,3 \%)$ a desarrollar procesos cognitivos superiores. Ello coincide con los resultados de este estudio acerca de las demandas de las preguntas analizadas en los libros de texto.

En el mismo estudio acerca de procesos cognitivos y funciones didácticas contenidas en los libros de texto de Ciencias Naturales, Meneses, Montenegro y Ruiz (2013) establecieron, en las conclusiones de su trabajo, que en los libros de texto se considera la exposición de conceptos y contenidos, la evaluación de lo aprendido y la construcción de conocimientos previos, los cuales se ubican en las categorías de recordar y comprender de la taxonomía de Anderson y Krathwohl (2001).

Meneses, Montenegro y Ruiz señalan que “[...] los textos escolares chilenos presentan menos oportunidades [...] para desarrollar habilidades y contenidos para aprender ciencias en comparación con Canadá y Singapur" (2013, p. 26). Las preguntas contenidas en los libros de texto de Ciencias Naturales de la Educación Primaria chilena no presentan diferencias estadísticamente significativas entre las preguntas formuladas entre los diferentes cursos estudiados.

Como perspectiva de continuidad, se puede señalar que otras investigaciones podrían reforzar el tema de la comprensión mediante la formulación de preguntas. Este es un mecanismo o estrategia que representa una vigorosa actividad metacognitiva (GAVELEK; RAPHAEL, 1985). Los libros de texto de primaria de Ciencias Naturales deberían incorporar más y mejores preguntas que apunten al desarrollo de dimensiones cognitivas superiores, con la finalidad de optimizar el aprendizaje de procesos científicos. Adicionalmente, los estudiantes podrían avanzar en su propio desarrollo cognitivo tal como la comprensión del mundo natural y social que los rodea y otras dimensiones más elevadas relativas a la aplicación, evaluación y creación. Por otra parte, los profesores podrían ampliar las formas de evaluación del aprendizaje y mejorar la transferencia mediante el desarrollo de procesos cognitivos superiores fomentados en los estudiantes.

Tanto en el nivel del razonamiento como de la dimensión del proceso cognitivo, no existen diferencias estadísticamente significativas entre las preguntas de los libros de texto en los diferentes cursos estudiados.

Las preguntas de los libros de texto corresponden a bajas demandas cognitivas y de razonamiento científico, lo cual inhibiría un mayor desarrollo intelectual de los estudiantes.

Estudiar las preguntas en los libros de texto de Ciencias Naturales es necesario, dado que hacer ciencia de los científicos no es lo mismo que hacer ciencia escolar. Sin embargo, para fomentar el pensamiento y quehacer científico en los estudiantes, se requiere emular el trabajo científico. Ello es posible, en mayor o menor medida, si se considera como base la formulación de preguntas, ya que el proceso de hacer ciencia siempre comienza con una o más interrogantes. La ciencia comienza con preguntas y, de acuerdo con Sanmartí y Oliveras (2011), la ciencia evoluciona porque la lectura de 
textos científicos forma parte de una práctica social. Así también se puede señalar, que la mente de los estudiantes se moldea gracias a la formulación de preguntas desafiantes y creativas en el ámbito de la ciencia.

\section{Referencias}

ANDERSON, Lorin; KRATHWOHL, David. A taxonomy for learning, teaching and assessing: a revision of Bloom's taxonomy of educational objectives. New York: Longman, 2001.

BLO0M, Benjamin et al. Taxonomy of educational objectives: the classification of educational goals. New York: Longmans, 1956.

CAMPANARIO, Juan Manuel; OTERO, José. La comprensión de los libros de texto. In: PERALES, Francisco Javier; CAÑAL DE LEÓN, Pedro (ed.). Didáctica de las ciencias experimentales: teoría y práctica de la enseñanza de las ciencias. Alicante: Marfil, 2000. p. 323-338.

CHILE. Ministerio de Educación. Política pública de textos escolares con recursos digitales complementarios. Santiago de Chile: Ministerio de Educación, 2015.

CHIN, Christine. Students' questions: fostering a culture of inquisitiveness in science classrooms. School Science Review, Hertfordshire, v. 86, n. 314, p. 107-112, 2004.

CHIN, Christine; CHIA, Li-Gek. Problem-based learning: using students' questions to drive knowledge construction. Science Education, Tallahassee, v. 88, n. 5, p. 707-727, 2004.

CHIN, Christine; OSBORNE, Jonathan. Students' questions: a potential resource for teaching and learning science. Studies in Science Education, London, v. 44, n. 1, p. 1-39, 2008.

CHINN, Clark A.; MALHOTRA, Betina A. Epistemologically authentic Inquiry in schools: a theoretical framework for evaluating inquiry tasks. Science Education, Tallahassee, v. 86, n. 2, p. 175-218, 2002.

CHOPPIN, Alain. Pasado y presente de los manuales escolares. Revista Educación y Pedagogía, Medellín, v. 8, n. 29-30, p. 209-229, 2001.

COLÁS, María Pilar. El libro de texto y las ilustraciones: enfoques y perspectivas en la investigación educativa. Enseñanza \& Teaching, Salamanca, v. 7, p. 41-49, 1989.

COSTA, Joan. Diez casos específicos. In: MOLES, Abraham; JANISZEWSKI, Luc (ed.). Grafismo funcional. Barcelona: CEAC, 1990. p. 197-227.

DÍAZ-LEVICOY, Danilo et al. Gráficos estadísticos en libros de texto chilenos de ciencias naturales. Revista Didasc@lia, Las Tunas, v. 7, n. 4, p. 75-96, 2016.

GAVELEK, James R.; RAPHAEL, Taffy. Metacognition, instruction, and the role of questioning activities. In: FORREST-PRESSLEY, Donna-Lynn; MACKINNON, Gordon; WALLER, Gary (ed.). Metacognition, cognition and human performance. Orlando: Academic Press, 1985. p. 103-136. 
GIL-PÉREZ, Daniel et al. La enseñanza de las ciencias en la educación secundaria. Barcelona: Horsori, 1991.

GRAESSER, Arthur; PERSON, Natalie. Question asking during tutoring. American Educational Research Journal, Washington, DC, v. 31, n. 1, p. 104-137, 1994.

GRAESSER, Arthur; OZURU, Yasuhiro; SULLINS, Jeremiah. What is a good question? In: MCKEOWN, Margaret G.; KUCAN, Linda (ed.). Threads of coherence in research on the development of reading ability. New York: Guilford, 2009. p. 112-141.

GRAESSER, Arthur; PERSON, Natalie; HUBER, John. Mechanisms that generate questions. In: LAUER, Thomas W.; PEACOCK, Eileen; GRAESSER, Arthur (ed.). Questions and information systems. Hillsdale: Lawrence Erlbaum, 1992. p. 167-187.

GRAY, Peter. Engaging students' intellects: the immersion approach to critical thinking in psychology instruction. Teaching of Psychology, Thousand Oaks, v. 20, n. 2, p. 68-74, 1993.

HALPERN, Diane F. Changing college classrooms: new teaching and learning strategies for an increasingly complex world. San Francisco: Jossey-Bass, 1994.

HERNÁNDEZ, Roberto; FERNÁNDEZ, Carlos; BAPTISTA, María del Pilar. Metodología de la investigación. México: McGraw-Hill, 2010.

HOFER, Barbara K.; PINTRICH, Paul R. The development of epistemological theories: beliefs about knowledge and knowing and their relation to learning. Review of Educational Research, Thousand Oaks, v. 67, n. 1, p. 88-140, 1997.

JUANDÓ, Josep; PÉREZ-CABANÍ, María Luisa. La evaluación de los resultados de aprendizaje. In: GONZÁLEZ LOSADA, Sebastián (ed.). Evaluación y calidad en la universidad. Huelva: Universidad de Huelva, 2010. p. 265-270.

MARBACH-AD, Gili; SOKOLOVE, Phillip G. Can undergraduate biology students learn to ask higher level questions? Journal of Research in Science Teaching, Malden, v. 37, n. 8, p. 854-870, 2000.

MÁRQUEZ, Conxita; ROCA, Montserrat. Plantear preguntas: un punto de partida para aprender ciencias. Revista Educación y Pedagogía, Medellín, v. 18, n. 45, p. 61-71, 2006.

MARTINEZ-BONAFÉ, Jaume. Políticas del libro de texto escolar. Madrid: Morata, 2002.

MENESES, Alejandra; MONTENEGRO, Maximiliano; RUIZ, Marcela. Calidad de textos escolares para aprender ciencias: habilidades, contenidos y lenguaje académico. Santiago de Chile: PUC, 2013. Pontificia Universidad Católica de Chile (Proyecto FONIDE No: F6111111).

NÄSSTRÖM, Gunilla; HENRIKSSON, Widar. Alignment of Standards and assessment: a theoretical and empirical study of methods for alignment. Electronic Journal of Research in Educational Psychology, Almería, v. 16, n. 3, p. 667-690, 2008. 
OCCELLI, Maribel; VALEIRAS, Nora. Los libros de ciencias como objeto de investigación: revisión bibliográfica. Enseñanza de las Ciencias, Barcelona, v. 31, n. 2, p. 133-152, 2013.

OLIVEIRA, Alandeom W. Improving teacher questioning in science inquiry discussions through professional development. Journal of Research in Science Teaching, Malden, v. 47, n. 4, p. 422-453, 2010.

OTERO, María; GRECA, María. Las imágenes en los textos de Física: entre el optimismo y la prudencia. Caderno Catarinense de Ensino de Física, Florianópolis, v. 21, n. 1, p. 37-67, 2004.

PÉREZ-CABANÍ, María Luisa; CARRETERO-TORRES, María Reyes. La promoción de estudiantes estratégicos a través del proceso de evaluación que proponen los profesores universitarios. In: MONEREO I FONT, Carles; POZO, Juan Ignacio (ed.). La universidad ante la nueva cultura educativa: enseñar y aprender para la autonomía. Madrid: Síntesis-ICE de la UAB, 2003. p. 173-190.

PÉREZ-CABANÍ, María Luisa; CARRETERO-TORRES, María Reyes; JUANDÓ, Josep. Formación de profesores y evaluación. IRAESO: un instrumento para reflexionar sobre la evaluación del aprendizaje. La evaluación auténtica en enseñanza universitaria: investigación e innovación. Barcelona: Edebé, 2009.

SANMARTÍ, Neus; OLIVERAS, Begonya. Llegir per aprendre y per desenvolupar el pensament critic: reflexions des de l'aprenentatge de las ciencias. In: ESCOBAR, Cristina; NUSSBAUM, Luci (ed.). Aprende en una altra llengua. Bellaterra: UAB, 2011. p. 71-93.

SCARDAMALIA, Marlene; BEREITER, Carl. Test-based and knowldege-based questioning by children. Cognition and Instruction, Mahwah, v. 9, n. 3, p. 177-199, 1992.

THIBAUT, Catalina; MEDRANO, Daniela, JIMÉNEZ, Ana. Evaluación en aula de textos escolares: ¿una estrategia posible? Estudios Pedagógicos, Valdivia, v. 38, n. 2, p. 243-257, 2012.

TRILLO, Felipe. Competencias docentes y evaluación auténtica: ¿Falla el protagonista? Perspectiva Educacional, Valparaíso, n. 45, p. 86-102, 2005.

TYNER, Kathleen. Alfabetización audiovisual: el desafío de fin de siglo. In: APARICI, Roberto (ed.). La revolución de los medios audiovisuales: educación y nuevas tecnologías. Madrid: La Torre, 1993. p. 171-197.

VAN ZEE, Emily H. Ways to foster teachers' inquiries into science learning and teaching. In: MINSTRELL, James Arthur; VAN ZEE, Emily $H$. (ed.). Inquiring into inquiry learning and teaching in science. Washington, DC: American Association for the Advancement of Science, 2000. p. 100-119.

VIDAL-ABARCA, Eduardo; GILABERT, Ramiro; ROUET, Jean-Francois. El papel de las preguntas intercaladas en los textos de ciencias. Tarbiya, Madrid, n. 36, p. 129-146, 2005.

Recibido en: 17.05 .2019

Revisado en: 09.04.2020

Aprobado en: 12.05.2020 
Alejandro Sepúlveda es Doctor en Educación por la Universidad Humanismo Cristiano. Académico del Departamento de Educación de la Universidad de Los Lagos, Osorno, Chile. Sus publicaciones son en el área de las Ciencias Naturales y la evaluación del aprendizaje.

Andrea Minte es Doctora en Educación por la Universidad de Concepción y de Estocolmo. Académica del Departamento de Educación de la Universidad de Los Lagos, Osorno, Chile. Investiga en el ámbito del currículum y los textos escolares.

Danilo Díaz-Levicoy es Doctor en Ciencias de la Educación por la Universidad de Granada. Académico de la Facultad de Ciencias Básicas de la Universidad Católica del Maule, Talca, Chile. Investiga en el ámbito de la Didáctica de la Matemática y la Estadística. 